\title{
Treatment of Polycystic Ovary Syndrome from the Theory of "Yigui Homology"
}

\author{
Xin Zhao', Mei Chen ${ }^{2 *}$, Nan $\mathrm{Li}^{2}$, Hongli $\mathrm{Zhu}^{2}$ \\ ${ }^{1}$ Shaanxi University of Traditional Chinese Medicine, Xianyang 712000, China \\ ${ }^{2}$ The Affiliated Hospital of Shaanxi University of Traditional Chinese Medicine, Xianyang 712000, China
}

\begin{abstract}
Traditional medicine believes that emotional, room labor and other factors lead to the lack of blood circulation after the weather, and the consumption of Yin blood is too much, which can lead to the deficiency of blood essence. Over time, the deficiency of menstrual blood can not nourish the kidney essence, leading to abnormal pregnancy. The liver is congenital for women, and the kidney is congenital. The disharmony of liver and kidney is the key factor of women's disease. Starting from the "homology of Yi and GUI", this paper analyzes the main etiology and pathogenesis of the disease from three aspects: deficiency of blood essence, imbalance of Zang and Xie, and Yin not controlling Yang. Following the principle of simultaneous treatment of liver and kidney, starting from the three methods of "regulating and tonifying liver and kidney, tonifying kidney and activating blood circulation, and balancing yin and Yang", the liver and kidney are reconciled, and pregnancy is sometimes the best choice. The main purpose is to benefit PCOS patients.
\end{abstract}

Key words: The liver and kidney are homologous; PCOS; TCM treatment

Publication date: May, 2021; Publication online: 31 May, 2021

*Corresponding author: Mei Chen, mmchen2009@,126.com

Polycystic ovary syndrome (PCOS) is an endocrine disease characterized by reproductive dysfunction. In modern medicine, PCOS is divided into the category of "rare menstruation" and "infertility". Referring to the TCM diagnosis and treatment of PCOS in recent 10 years, it is found that TCM has unique advantages in the diagnosis and treatment of $\mathrm{PCOS}^{[1]}$. Based on the theory of visceral manifestation, the author tries to analyze the etiology, pathogenesis, diagnosis and treatment of PCOS from the theory of liver kidney homology, in order to improve the clinical efficacy of PCOS.

\section{Tracing the origin of the theory of "liver and kidney have the same origin"}

Liver kidney homology, also known as "Yigui homology", "blood essence homology". It is pointed out in Neijing that "kidney produces bone marrow, and marrow produces liver." This reflects the complementary relationship between liver and kidney in physiology and pathology, and lays the foundation for the theory of "liver and kidney homology";
"Wuqiguluru pill" was first recorded in Neijing. It is pointed out in Yizong must read that "tonifying the kidney means tonifying the liver It is the first time to put forward that "Yigui has the same origin and the liver and kidney are treated together".

\section{Exploration of the origin of PCOS along the "liver kidney homology"}

\subsection{The foundation of PCOS -- deficiency of blood} essence

It is pointed out in Zhang Shi Yi Tong that: "Qi is not consumed, but essence comes from kidney; If the essence does not leak, it will return to the liver and clear the blood. The liver stores blood, the kidney stores essence, and the essence and blood are mutually biochemical. The essence of the kidney is sufficient to transform the blood of the liver, to fill the blood, to fill the blood of the liver, to feed back the essence of the kidney, so that the blood of the liver and the essence of the kidney are both prosperous and harmful. Modern women take thinness as beauty. They control their 
diet, lose nourishment of the spleen and stomach, or dirty rooms, mental pressure and so on, which lead to the loss of nourishment of the kidney essence, and lead to the lack of liver and blood; Or the woman because of excessive consumption of Yin blood, lack of blood essence, kidney injury, so the lack of blood. As the old saying goes: "Eight vessels belong to the liver and kidney", the liver, blood and kidney essence nourish Chong Ren. If it is insufficient, it will not fill Chong Ren, and the sea of blood will be full and full, resulting in less menstruation, amenorrhea and even infertility.

\subsection{The key of PCOS}

"Yizong Jinjian" says that "the kidney is hard to store, more deficiency and less excess, because the son of ganmu prefers to dredge the mother Qi". The kidney is in charge of closing and storing, and the liver is in charge of dispersing and discharging. Liver Qi dispersing prevents the opening and closing of kidney qi too much, and the kidney qi is closed to keep the degree of liver Qi dispersing; They cooperate with each other to maintain the balance of Qi, blood, yin and Yang in the body. Emotional disorders hurt the liver, liver laxation is not enough, resulting in kidney too sealed, loss of opening and closing, resistance Chong Ren, Chong Ren stagnation, blood sea deficiency, delayed menstruation, amenorrhea and other diseases. The function of dispersing and sealing of the liver and kidney is out of balance, and the water and blood in the body are abnormal. Chong Ren two pulse congestion, the uterus can not be full and overflow, abnormal menstrual water; Chong Ren barrier, eggs can not be Yin blood feeding and gradually mature, or ovulation disorders, even infertility.

\subsection{The pivot of PCOS}

Chen Xiuyuan recorded in essentials of women's studies: "Those who do not adjust because of the water It is caused by the multiplication of yin and Yang. Due to congenital deficiency, deficiency of the liver and kidney, or excessive labor, damage to the liver and kidney, and then damage to the liver and kidney yin, if the liver yin deficiency can be down to the kidney yin, make the kidney yin deficiency, kidney yin deficiency cannot nourish the liver wood, lead to liver yin deficiency, so the Yin of the two viscera Yin liquid are all deficient, cause Yin not control Yang, damage to the uterus cell pulse, lead to kidney Tiangui Chongren. The adjustment function of uterine axis is abnormal, so irregular menstruation occurs, even infertility.

\section{3 "Yigui homology"' guiding to the diagnosis and treatment of PCOS}

\subsection{Tonifying liver and kidney, nourishing essence and blood}

True story of Medicine: "Cover Chong Ren's blood, liver controls it."; There is also "the water comes from the kidney", so the liver and kidney are out of tune, and the essence and blood are out of nourishment, which are the main pathogenic factors of the disorder. TCM diagnosis and treatment takes tonifying the liver and kidney as the method, aiming at harmonizing the liver and kidney, regulating the essence and blood, enriching the sea of blood, and keeping the moon on time. This disease is mainly due to deficiency and excess, so the main medicine of tonifying liver and kidney is added with the products of promoting blood circulation and removing blood stasis, resolving phlegm and manic dampness. Professor Zhu Nansun used the method of tonifying liver and kidney to regulate PCOS, and the patient finally achieved the long cherished wish of pregnancy ${ }^{[2]}$. Zhang Cuixiang summarized Cai Lianxiang's clinical experience and concluded that Tiaojing Zhuyun decoction had a good prognosis in the treatment of PCOS patients ${ }^{[3]}$.

\subsection{Tonifying kidney and soothing liver, regulating Zang Xie}

According to the clinical guide medical records: "In women, the liver is congenital. If the liver Qi is stagnant, the blood is Qi stagnation, the Chong Ren is not smooth, and the sea of blood is not full, then it leads to less menstruation." Liver depression damages the kidney, resulting in deficiency of both liver and kidney. The therapy of soothing the liver and tonifying the kidney aims at regulating the liver and kidney, storing and reducing the order, and getting pregnant. Ling Xiping used Shugan Bushen Decoction to treat PCOS with gestational disorder, and achieved good results ${ }^{[4]}$. Professor Zhao hengxia used the method of soothing the liver and tonifying the kidney to treat PCOS patients with sparse menstruation and obesity. After several cycles of diagnosis and treatment, the menstruation was normal ${ }^{[5]}$.

\subsection{Nourishing kidney and liver, balancing yin and yang}

Ye Tianshi pointed out: "The eight meridians are beautiful and belong to the liver and kidney." When the channels are smooth, the blood will flow smoothly and the sea of blood will be rescued. When the water is flowing, the follicle will be discharged smoothly. Clinically, nourishing liver and kidney, regulating qi and regulating menstruation are 
commonly used. Its core is to nourish the liver and kidney yin, that is, yin and Yang and water. Professor Tan Yong uses nourishing yin and nourishing yang to help PCOS patients regroup menstrual cycle and ovulate again ${ }^{[6]}$. Professor Dai Deying used the method of tonifying kidney and nourishing yin to treat PCOS in clinical practice, and the curative effect is very $\operatorname{good}^{[7]}$.

\section{Conclusion}

Under the guidance of traditional Chinese medicine theory, this paper analyzes the etiology and pathogenesis of PCOS, and discusses the diagnosis and treatment of PCOS, hoping to provide ideas for clinical diagnosis and treatment, and also provide reference for the treatment of other diseases.

\section{References}

[1] Zhang L, Tao YL. Research progress on treatment of polycystic ovary syndrome with traditional Chinese medicine $[\mathrm{J}]$. Journal of Gansu University of Traditional Chinese Medicine, 2015, 32 (4): 75-78.

[2] Jia M, Xu LW, Zhang TT, et al. Professor Zhu Nansun's flexible use of "tonifying liver and kidney, dispersing Li, Chong Ren" method in the treatment of female infertility $[\mathrm{J}]$. Sichuan Traditional Chinese Medicine, 2013, 31(8): 122-125.

[3] Zhang CZ, Cai LX. Application of CAI Lianxiang's "attaching importance to essence and blood" in gynecological clinical prescriptions [J]. Chinese Journal of Traditional Chinese Medicine, 2016, 31(2): 516-518.

[4] Ling XP. Clinical observation of Shugan Bushen Decoction in the treatment of polycystic ovary syndrome infertility $[\mathrm{J}]$. Bright Chinese Medicine, 2020, 35(1): 64-67.

[5] Zhang Z, Zhou DC, Liu Y, et al. Zhao Hengxia's experience in treating obese polycystic ovary syndrome [J]. New Chinese Medicine, 2019,51

[6] Mao M, Tan Y. Professor Tan Yong's experience in treating polycystic ovary syndrome with sequential therapy of nourishing yin and tonifying yang traditional Chinese medicine [J]. Chinese Medicine Guide, 2015, 21(11): 94-96.

[7] Yang BY, Zhou YC, Zeng WW, et al. Dai Deying treats polycystic ovary syndrome [J]. Journal of Changchun University of Traditional Chinese Medicine, 2019, 35(2): $239-241+245$. 